\title{
Optimized Frequency Selective Surface for the Design of Magnetic Type Thin Broadband Radio Absorbers
}

\author{
V. Babayan ${ }^{a, *}$, N.E. Kazantseva ${ }^{a}$, Yu.N. KazAntsev ${ }^{b}$, J. VilČÁKová ${ }^{a}$ And R. MouČKa ${ }^{a}$ \\ ${ }^{a}$ Centre of Polymer Systems, Tomas Bata University in Zlin, Tr. T. Bati 5678, Zlin, 760 01, Czech Republic \\ ${ }^{b}$ Fryazino Branch of Kotel'nikov Institute of Radio Engineering and Electronics, Russian Academy of Sciences, \\ pl. Vvedenskogo 1, Fryazino 141190, Russia
}

\begin{abstract}
A novel frequency selective surface for expansion of operating frequency range of magnetic-type radio absorbers was elaborated. Frequency selective surface represents an array of flat electrically conductive elements deposited on the polyethylene terephthalate (PET) foil. The elements have a closed-loop structure on the one side of the foil, and on the opposite side, the conductive elements have the shape of solid fragments which connect projections of the neighboring closed loops. The advantage of such frequency selective surface design is that owing to capacitive shunting one can achieve the value of frequency selective surface quality factor ( $Q$-factor) as low as 0.5 . Operating frequency range and thickness of designed radio absorbers depend on the composition of polymer-magnetic material and the position of frequency selective surface therein.
\end{abstract}

DOI: 10.12693/APhysPolA.131.1147

PACS/topics: 41.20.-q

\section{Introduction}

Nowadays, the solution of problems associated with electromagnetic interference and the negative impact of electromagnetic radiation on human beings are of particular importance due to the rapid penetration of electronics to virtually all fields of a modern life. One of the means to address these concerns is the use of radio absorbers (RAs). For the optimal design of RAs the reduction of weight and thickness as well as broadening of operating frequency range of absorber must be considered simultaneously $[1,2]$. The reflection coefficient $(R)$ is a parameter that characterizes the absorbing ability of RA in decibels (dB). The level of $R$ equal to $-10 \mathrm{~dB}$ insures $90 \%$ absorption of incident energy by RA (presuming the absence of transmitted energy). The operating frequency range, which is another important parameter characterizing RA, is the difference between the edge frequencies $\left(f_{\max }\right.$ and $\left.f_{\min }\right)$ of the interval where $R$ is below $-10 \mathrm{~dB}$. Any RA operates in a limited operating frequency range. One of the methods for expanding the bandwidth of RA without increasing its thickness is the incorporation of frequency selective surface (FSS) into the structure of RA [2-4]. FSS consists of a dielectric plate which usually comprises periodically spaced planar conductive elements. Elements are made in various shapes, namely solid squares, loops, etc. Mechanism of expansion of frequency band by the incorporation of FSS in the radio absorbing material is related to change of the frequency dependence of effective thickness of an absorber by introducing additional phase shifts to a transmitting wave. Elements reflect the incident wave according to their shape,

*corresponding author; e-mail: babayan@cps.utb.cz periodicity and the dielectric property of a substrate.

Generally, the width of operating frequency range of RA depends on resonance frequency and $Q$-factor of FSS as well as on its position in absorber. As a rule, the lower the value of $Q$-factor the wider will be an operating frequency range of RA. However, the realization of FSS with a $Q$-factor lower than 1.3 with the aid of productive and low-cost technology is rather challenging. Having this fact in mind, we came up with a novel FSS the unique structure of which allows one to achieve the value of a $Q$ factor as low as 0.5.

The effectiveness of FSS application in RAs operating in microwave region was successfully verified.

\section{Experimental}

The FSS was printed on $100 \mu \mathrm{m}$ thick PET substrate using inkjet FUJIFILM Dimatix Materials Printer DMP2800 series. The ink used was based on highly concentrated dispersion of stabilized silver nanoparticles.

Polymer-magnetic composites where FSS was incorporated for the design of RAs had the following compositions:

Composite 1: 50 vol.\% of magnetic filler (Carbonyl iron (CI) ES grade, BASF, Germany), 50 vol.\% of silicon elastomer SYLGARD 184 (Dow Chemical, USA).

Composite 2: 40 vol.\% of magnetic filler (CI HQ grade), $45 \mathrm{vol} . \%$ of silicon elastomer SYLGARD 184, $15 \mathrm{vol} . \%$ of MSVP A9 glass microspheres (NPO Stekloplastik, Russia) which were used to improve the homogeneity of the CI particle distribution in the composite, as well as to control its complex permittivity.

Composite 3: 30 vol.\% of magnetic filler (CI HQ grade), $50 \mathrm{vol} \%$ of silicon elastomer SYLGARD 184, $20 \mathrm{vol} . \%$ of MSVP A9 glass microspheres.

The composite sheets were manufactured via compression molding technique. The components were mixed 
in the required proportion to give a homogeneous mixture. Then, the mixture was poured into the mold, placed on the heated press platen and cured for four hours at temperature of $80^{\circ} \mathrm{C}$. The frequency dependence of complex magnetic permeability and dielectric permittivity of composites were studied using Agilent E4991A Impedance/Material Analyzer and Agilent N5230A PNA-L series two-port vector circuit analyzer (Agilent Technologies, USA).

\section{Results and discussion}

The schematic representation of the manufactured FSS is shown in Fig. 1.
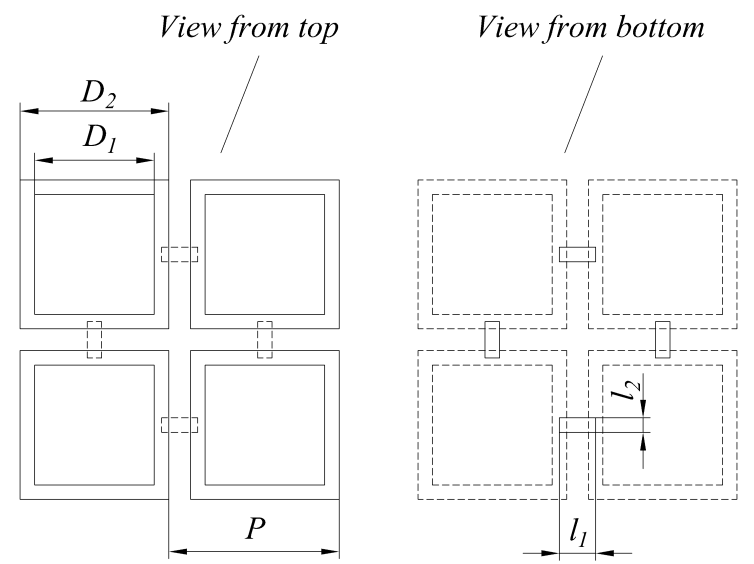

Fig. 1. Schematic representation of FSS.

As can be seen, the FSS is formed by the array of square loops (main grate) which are deposited with the certain periodicity on the one side of the PET substrate and solid rectangular fragments which connect the projections of square loops on the opposite side of PET foil (secondary grate). In such construction, the solid rectangular fragments play the role of capacitive shunts between the neighboring elements of the main grate. The effect of the capacitive shunts on the resonance frequency and $Q$-factor of FSS is similar to the effect of minimizing the distance between the square loops $\left(P-D_{2}\right)$ since both lead to the increase of the effective capacitance between the loops. However, the disadvantage of the second method is that the realization of grate where the distance between adjacent square loops is smaller than $0.15 \mathrm{~mm}$ with the aid of productive and low-cost technology is rather challenging.

The $Q$-factor of the FSS with optimized parameters $\left(D_{1}=8 \mathrm{~mm}, D_{2}=10.5 \mathrm{~mm}, P=11 \mathrm{~mm}, l_{1}=2 \mathrm{~mm}\right.$, $l_{2}=1 \mathrm{~mm}$ ) was calculated based on the experimentally measured frequency dependence of reflection coefficient from FSS surface in free space (Fig. 2).

The value of $Q$-factor as low as 0.5 was achieved while the distance between adjacent square loops was $0.5 \mathrm{~mm}$.

The effectiveness of application of such FSS in the design of RAs is demonstrated on the example of metalbacked RAs based on composites 1, 2, and 3 which are

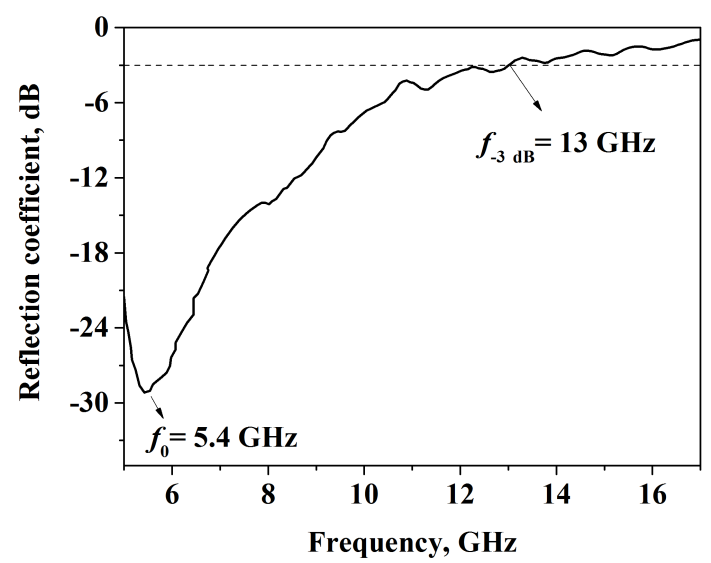

Fig. 2. Frequency dependence of reflection coefficient from FSS surface measured in free space [5].

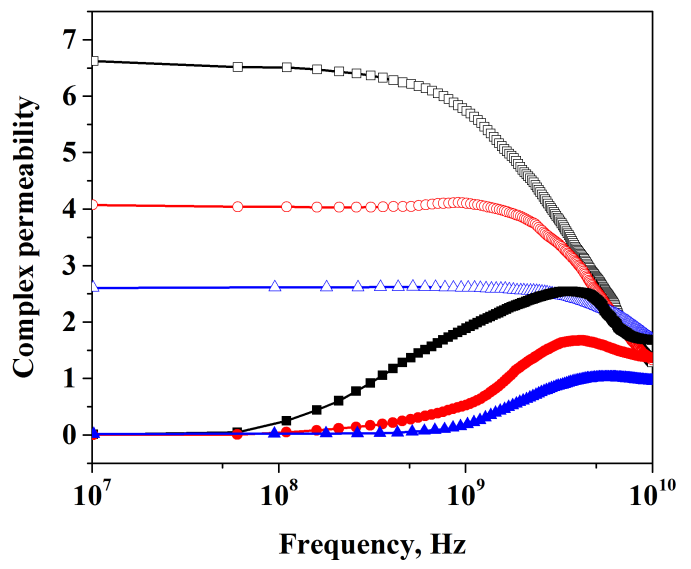

Fig. 3. Frequency dependence of complex magnetic permeability of composites 1,2 and 3. Curves with open and solid symbols represent the real and imaginary parts of permeability, respectively ( $\square$ - composite 1 , ○- composite 2, $\Delta \boldsymbol{\Delta}$ - composite 3) [5].

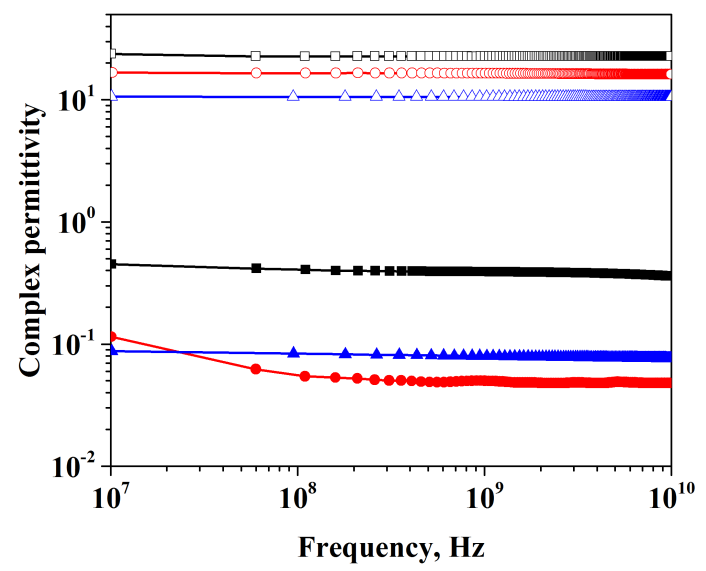

Fig. 4. Frequency dependence of complex permittivity of composites. Curves with open and solid symbols represent the real and imaginary parts of permeability, respectively (ם- composite $1, \circ \bullet$ - composite 2 , $\Delta \boldsymbol{\Lambda}$ - composite 3) [5]. 
TABLE I

Electrodynamic characteristics of RAs based on the composites 1,2 , and 3 .

\begin{tabular}{c|c|c|c|c|c}
\hline \hline RA & FSS & $d[\mathrm{~mm}]$ & $f_{\min }[\mathrm{GHz}]$ & $f_{\max }[\mathrm{GHz}]$ & $f_{\max } / f_{\min }$ \\
\hline \multirow{2}{*}{1} & - & \multirow{2}{*}{3} & 1.8 & 2.9 & 1.6 \\
& $\checkmark$ & & 1.4 & 4.0 & 2.9 \\
\hline \multirow{2}{*}{2} & - & \multirow{2}{*}{2} & 3.6 & 6.5 & 1.8 \\
& $\checkmark$ & & 3.0 & 8.3 & 2.8 \\
\hline \multirow{2}{*}{3} & - & \multirow{2}{*}{1.5} & 7.6 & 13.1 & 1.7 \\
& \multirow{2}{*}{$\checkmark$} & & 6.0 & 16.9 & 2.8
\end{tabular}
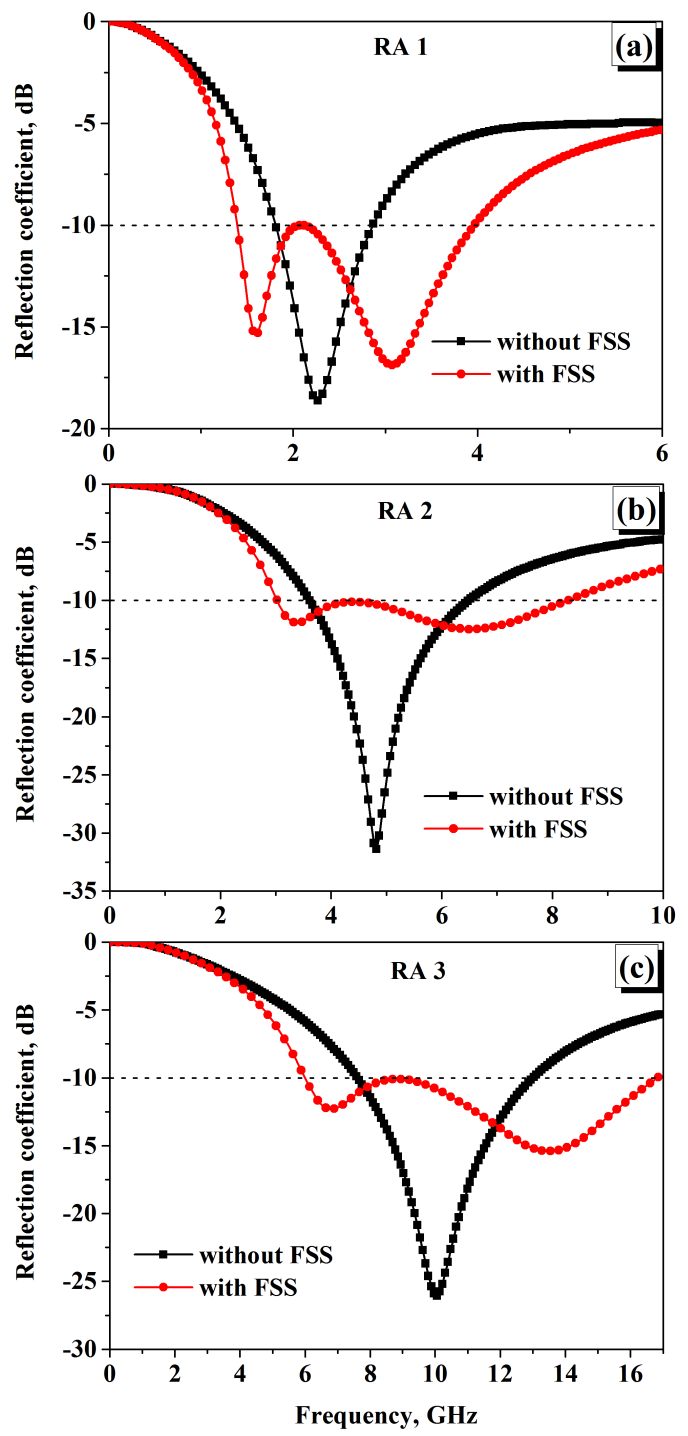

Fig. 5. Calculated frequency dependence of reflection coefficient of RAs based on composites 1, 2, and 3 [5].

named RA 1, RA 2, and RA 3, respectively. The calculation of the frequency dependence of reflection coefficient from the surface of RAs was performed via transmission line theory $[2,6]$ using experimentally measured complex permeability (Fig. 3) and permittivity spectra (Fig. 4) of composites 1,2 , and 3 . We considered the case when the
FSS is embedded into composite structure at depth equal to $2 / 3$ of composite layer thickness calculated from the side of electromagnetic wave incidence, since it has been shown in our patent that this depth ensures the maximum bandwidth of RA [5]. As can be seen from Table I and Fig. 5 incorporation of FSS into the RA structure substantially broadens the operating frequency range of all considered RAs without increase of their thickness. The extension of bandwidths by $81 \%, 56 \%$ and $65 \%$ is observed for RA 1, RA 2, and RA 3, respectively. After incorporation of an FSS into the structure of the RA, the shape of the frequency dependence of the reflection coefficient is transformed from usual resonant-type to twohumped. Extension of the operating range of the RA with FSS can be explained by the interaction between the resonance in the FSS and the resonance in the polymermagnetic composite under the condition of closeness of resonance frequencies. In this case, as shown in [3], the range in which the RA is matched with free space is substantially extended.

\section{Conclusions}

A novel FSS with $Q$-factor as low as 0.5 was elaborated. Such low value of $Q$-factor is achieved owing to the unique design of FSS where the effect of capacitive shunting among the elements of the main and secondary grates of FSS is successfully utilized. The efficiency of the FSS application to broaden the bandwidth of magnetic-type RAs operating at microwave band was demonstrated. The extension of bandwidths of three considered RAs by $56 \%, 65 \%$, and $81 \%$ without an increase in RAs thickness was achieved.

\section{Acknowledgments}

This work was supported by the Ministry of Education, Youth and Sports of the Czech Republic - Program NPU I (LO1504).

\section{References}

[1] K.N. Rozanov, IEEE Trans. Antennas Propag. 48, $1230(2000)$.

[2] Y.N. Kazantsev, A.V. Lopatin, N.E. Kazantseva, A.D. Shatrov, V.P. Mal'tsev, J. Vilcakova, P. Saha, IEEE Trans. Antennas Propag. 58, 1227 (2010).

[3] A.V. Lopatin, Y.N. Kazantsev, N.E. Kazantseva, V.N. Apletalin, V.P. Mal'tsev, A.D. Shatrov, P. Saha, J. Commun. Technol. Electron. 53, 1114 (2008).

[4] J. Yang, Z.X. Shen, IEEE Antennas Wirel. Propag. Lett. 6, 388 (2007).

[5] N.E. Kazantseva, Y.N. Kazantsev, V.A. Babayan, R. Moucka, J. Vilcakova, P. Urbanek, I. Kuritka, CZ Patent, 305905 (2016).

[6] V.A. Babayan, Y.N. Kazantsev, A.V. Lopatin, V.P. Mal'tsev, N.E. Kazantseva, J. Commun. Technol. Electron. 56, 1357 (2011). 